J. Comp. Int. Sci. (2015) 6(3):91-98

http://epacis.net/jcis/PDF_JC/S/JC/S-0098.pdf

jcis@epacis.net

@2015 PACIS (http://epacis.net)

\title{
Using system dynamics to simulate the stellar nucleosynthesis and galactic evolution
}

\author{
Orlando J. Katime Santrich ${ }^{a, b 1}$, Carlos J. Barrios Hernández ${ }^{c, d}$ and Hugo H. Andrade Sosa ${ }^{d}$ \\ ${ }^{a}$ Centro Halley de Astronomía y Ciencias Aerospaciales, Universidad Industrial de Santander UIS, \\ Bucaramanga, Colombia. \\ ${ }^{b}$ Coordenação de Astronomia e Astrofísica, Observatório Nacional ON, Rio de janeiro RJ, Brazil. \\ ${ }^{c}$ Centro de Supercomputación y Cálculo Científico, Universidad Industrial de Santander UIS, \\ Bucaramanga, Colombia. \\ ${ }^{d}$ Grupo de Modelamiento y Simulación SIMON, Universidad Industrial de Santander UIS, Bucaramanga, Colombia. \\ Received on Sep 22, 2015 / Accepted on Dec 31, 2015
}

\begin{abstract}
System dynamics is a methodology to model and simulate complexity in science and engineering. In this approach there are limited mathematical descriptions due to a large time in the behavior and a nonmathematical definition of the relationships between variables of the phenomena. It proposes causality and feedbacks to explain relationships between components. In spite that there are many examples of complex systems in Astrophysics, the uses of System Dynamics in this area are not documented. However, we used this methodology to develop models of the stellar nucleosynthesis on the main sequence and galactic evolution to exploit the possibility to treat important aspects as complexity, scale factors, unknown elements and known variables in the same context. For nucleosynthesis reactions, feedback loops keep the solar-type stars on the main sequence and production of chemical elements. The galactic evolution model has two feedback loops connected through density of molecular gas, generating the dynamic of stellar formation. Our results for energy emission rate, nuclear luminosity and central and effective temperatures for a solar-type star are similar to those found in the literature. As well as the qualitative behavior of the stellar mass, molecular gas density and intergalactic gas density for the galactic evolution model. Therefore we concluded that System Dynamics is a powerful tool for modelling complex phenomena with feedback in Astrophysics.
\end{abstract}

Keywords: system dynamics; stellar nucleosynthesis; galactic evolution; computational astrophysics.

\section{Introduction}

System Dynamics (SD) arises in the 60's at the Massachusetts Institute of Technology, led by Jay Wright Forrester, to apply the concept of feedback control theory to understand the complexity of phenomena in the industry (Forrester, 1961). Over the years, the SD has been expanding its scope from physical phenomena to social phenomena, through the complex problems of urban dynamics (Forrester 1969), business (Sterman, 2000), education (For-

\footnotetext{
${ }^{1}$ E-mail Corresponding Author: osantrich@on.br
}

rester, 1994), chemical reactions (Andrade et al. 1994), environmental sciences (Ford, 1999) and global change among others. In addition, the SD in its versatility is seen as a modeling technique (Forrester, 1961), a methodology (Ford, 1999) or as a systemic dynamic language paradigm (Andrade et al. 2001). The SD is an alternative to account for the dynamic complexity of phenomena explicable in terms of feedback loops, systems in the modeling process can be approximated by linear or nonlinear differential equa- 
tions; as appropriate, with the complement of fuzzy logic, neural networks or other mathematical tools of knowledge representation. The methodology facilitates the modeling process using two basic tools: influence and state variable rate diagrams. The influence diagram is a scheme where the dynamical behaviour is confirmed and its mathematical representation is the state variable-rate diagram. SD demands computational frameworks to associate diagrams, rates, state variables and relationships among components in a system. There are different frameworks knowing for modeling and simulation community, from the use of framework based in MATLAB (as SIMULINK), Wolfram Mathematica, COMSOL until in-house scientific tools as Dynaplan or EVOLUTION $^{2}$. We use this last framework, proposed by the SIMON Group in Modeling and Simulation Research at Universidad Industrial de Santander, Bucaramanga, Colombia.

System dynamics proposes an approach for the treatment of high complexity to understand the behavior of systems and to facilitate the modeling and simulation. It deals with feedback loops and time delays that affect the entire system involving some external factors. Taking the advantage of feedback loop's treatment and the complexity's treatment in a high level, we propose the use of SD on the modeling of astronomical phenomena. If the phenomenon is understanding in terms of its components, a description of its dynamical behavior is established using feedback. Then, the feedback is a consequence of the causal co-relationships among the elements of the system (System Thinking. Forrester 1987). Because of fact that SD belongs to the System Thinking (ST), which is a way of understanding every phenomenon in terms of its components, it establishes the dynamical explanation of the system on accounting the feedback, being a result of causal co-relationships between the elements of the system. Therefore, it identifies and analyzes the elements and its interconnections to describe the behavior of the system as a function of these. In order to show how does it is possible to use system dynamics in Astronomy to build mathematical models of physical complex systems, two cases will be presented: Proton Proton Chain (PPC) and galactic evolution process. PPC is the stellar nucleosynthesis process that occurs in solar-type stars on stability's phase called main sequence.

Although there are many examples of dynamical systems with feedback in Astronomy, there are no references of mathematical modelling using SD. The article is presented as follows: the second section shows the principles to use SD in Astronomy; third section expresses the astrophysical concepts and modeling process for PPC; forth section is similar but for the galactic evolution process and the fifth section we finish with our conclusions about the use of SD in Astronomy and present perspectives.

\section{System dynamics as modeling and simu- lation tool}

Complexity demands the use of computer tools following methodological statements. The proposal of implementing SD as a methodological tool for the construction of mathematical models of astrophysical phenomena with feedback loops is discussed in this section. Features in Astronomy such as time scales, large scales of the involved systems, understood and non-understood process in the same context and unknown mathematical relationship between variables, are interesting to make the astrophysical phenomena as good candidates to be modeled and simulated with SD. These reasons can be summarized as following:

- Feedback loops and dynamical behavior: the feedback loops or feedback refers to the situation where a certain variable $\mathrm{X}$ affects variable $\mathrm{Y}$ and vice versa, producing a series of effects. So, interaction X-Y cannot be studied independently, only the study of both interactions as a whole will lead us to the correct results. Therefore, feedback is the generator of dynamical behavior observed in the system/phenomenon. According to ST (Forrester, J. 1986, 1987), if a phenomenon is understood in terms of its components, a dynamical description of its behavior is established using feedback. In another words, feedback loops are consequence of the causal co-relationships between the elements of the system showing the complexity involved. In Physics and Astronomy there are many examples of systems/phenomena where such feedback is present. The two cases studied in this work are dynamical systems where feedback appears in its description and corresponds with definition of dynamical system given by SD: a set of interacting parts that constitute a whole with feedback loops (Forrester, J. 1992). Because of the special attention that ST puts in feedback loops, it makes possible to simulate

\footnotetext{
${ }^{2}$ More tools and frameworks can see in http://tools.systemdynamics.org/
} 
and model the dynamical behavior and, therefore, to improve the understanding of the complexity. Usually feedback loops involving all variables in the system and therefore all the influence relationships and dynamical interpretations are unveiled.

- Scales: usually in Astrophysics the time scales are large, of the order of million years. We can cite some examples: the solar scale age, of the order of $4.5 \times 10^{9} \mathrm{Yr}$; the oldest stars in Milky Way are $13.0 \times 10^{9} \mathrm{Yr}$, values very near to the age of the Universe found through different methods $13.4 \times 10^{9} \mathrm{Yr}$ (Ade, P. 2015). These large times are obviously outside of the human scale and are an additional problem for interpretation of the observations. Furthermore, in several astrophysical situations there are big differences in the time scales into the same phenomenon. One classic example is the PPC with reactions at time scales of million years and also others of few seconds. A similar situation happens with another physical quantities like masses, sizes and distances. The difference of scales within the same system present in Astronomy makes it difficult for any process of computational simulation and even to interpret the observations. However, because of SD representations like state variables, rates, exogenous variables and delays, the process of modeling and simulation for these systems is remarkably facilitated.

- Unknown processes and non-mathematical descriptions: in several astrophysical situations, there are some partially unknown variables that have correlations with another known variables into the same phenomenon or system. The partially and totally unknown variables present in the phenomenology of complex systems are a consequence of non-mathematical relationships between the variables and the nonmathematical descriptions are the main barrier to understand complexity of the phenomenon. This situation is unveiled through influence relationships, which are obtained from the ST's concepts and provided by SD's representations. One example of this situation is the galactic evolution. As it will be shown in the Section 4 , the evolution process of the Milky Way has two feedback loops, the variables describing the loop-2 are unknown, or less known, relative to the variables in loop-1.

The construction of Influence Diagram (ID) is based on theoretical and observational assumptions of the phenomenon. The ID identifies the relationships between different variables through arrows showing how each variable affects the other forming reinforcing feedback loops and delays positive or negative. It helps to find the feedback loops and therefore to confirm the dynamical behavior of the system. The second step is to build the state variable - rate diagram frequently called Forrester Diagram (FD), this diagram is built from SD's representations, representing linear or nonlinear differential equations. The correlation state variable rate is illustrated in Figure 1.

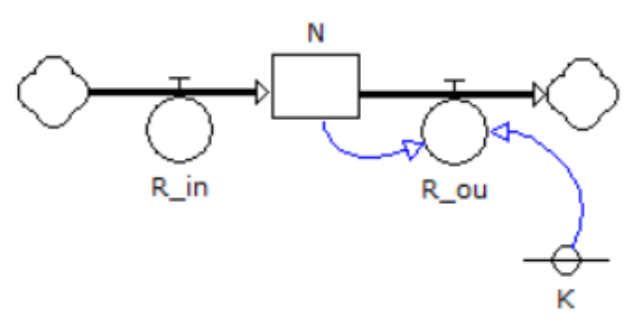

Figure 1 - State variable rate correlation. The state variable is represented by square while the inflow and outflow rates are represented by circles. The small circle crossed by vertical line represents a constant.

The correlation represented in Figure 1 correspond to the differential equation:

$$
\frac{d N}{d t}=R_{\text {ou }}(t)-R_{\text {in }}(t),
$$

where $N$ is the state variable, $R_{i n}$ and $R_{o u}$ are the inflow and outflow rates, respectively. $R_{o u}$ depends on the state variable, i.e. $R_{o u}(N, k)$, being $\mathrm{k}$ a constant. The number of correlations state variable - rate in the $\mathrm{FD}$, determines the set of differential equations that describes the dynamical behavior of the system.

The previous construction of the Influence Diagram is necessary because in majority of the cases, the complexity become so high to identify the feedback loops directly in the FD, which may lead to errors and possibly not to find important correlations. The final step using SD is to obtain the mathematical model from FD's differential equations and solve it numerically under different simulation conditions.

In addition to state variables, rates and variables; SD approach offers others representations such 
as constants, delays, exogenous variables and nonlinearities. The meaning of each symbol is explained in Figure 2.

\begin{tabular}{|l|l|}
\hline & State variable: physical quantity describes the state of the system. \\
\hline & Rates: outflow and inflow rates of the State Variable. \\
\hline & Variable: it represents a variable physical quantity. \\
\hline & Exogen Variable: it describes medium's action on the system. \\
\hline & Delay: it represents delay time between variables in the system. \\
\hline
\end{tabular}

Figure 2 - Representations commonly used in the SD approach.

\section{Modeling the stellar nucleosynthesis}

Stellar nucleosynthesis process happens in some phases of the stellar evolution, the main sequence represents the stability for the stars. PPC occurs in solar-type stars, our Sun is considered as a example of low-mass star. In fact, the Sun is an astrophysical laboratory where all theories for stellar evolution are proved and its values like mass, radius, age between others are taken into account as a reference. We have considered low-mass stars in the range of $(0.08-2.50) M_{\odot}$ (Catelan, M. 2006). Being a thermonuclear reaction, PPC is highly dependent on central temperature which in turn depends on the internal density. This reaction is presented like dynamical system because the elements like Protons collide with each other forming the rest of the elements of the reaction; the feedback loops produced by these interactions maintain the dynamics in the stellar interior. This feature correspond to the definition of dynamical system given by System Dynamics: a set of interacting parts that constitute a whole with feedback loops. PPC has three branches called PPC I, II and III. The first branch is the most important because it occurs $85 \%$ of the time into the Sun, PPC II occurs $14 \%$ and PPC III 1\% (see Schwarszchild, M. 1955; Parker, P. et al. 1964; Clayton, D. 1975; Kippenhahn, R., et al. 2012). The equations for PPC I \& II are given by:

$$
\begin{gathered}
\mathrm{H}+\mathrm{H} \rightarrow{ }^{2} \mathrm{H}+e^{+}+\nu \\
{ }^{2} \mathrm{H}+\mathrm{H} \rightarrow{ }^{3} \mathrm{He}+\gamma
\end{gathered}
$$

$$
\begin{gathered}
{ }^{3} \mathrm{He}+{ }^{3} \mathrm{He} \rightarrow \mathrm{H}+\mathrm{H}+{ }^{4} \mathrm{He} \\
{ }^{3} \mathrm{He}+{ }^{4} \mathrm{He} \rightarrow{ }^{7} \mathrm{Be}+\gamma \\
{ }^{7} \mathrm{Be}+e^{-} \rightarrow{ }^{7} \mathrm{Li}+\nu e \\
{ }^{7} \mathrm{Li}+{ }^{1} \mathrm{H} \rightarrow{ }^{4} \mathrm{He}+{ }^{4} \mathrm{He}
\end{gathered}
$$

In Figure 3, we show the feedback loops between the elements to confirm the dynamical character of the PPC. This figure is built based on equations (2)(7).

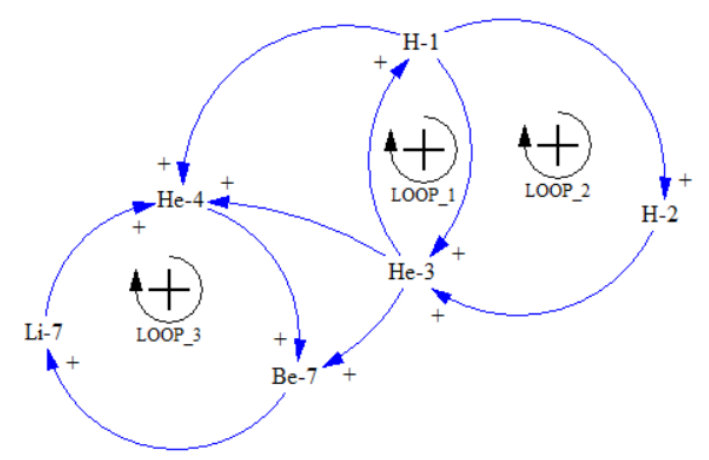

Figure 3 - Influence diagram for PPC I \& II. This is the first modeling step with SD. LOOP_1, LOOP_2 and LOOP_3 are the feedback loops developed from equations (2) to (7) and they are positive loops.

Coming to the second step of modeling, PPC's FD is shown in Figure 4. The state variables (H11, H12, H13, H14, H21, H22, He31, He32 \& He4) represent the number density of particles inside the stellar core whereas the thermonuclear rates $\mathrm{R}$ (with subscripts) represent the collision frequencies between the particles. In this work we have modeled PPC I to be the reaction with more frequency.

From FD we have obtained the mathematical model, i.e. the equations (8)-(12). Here, equation (8) represents the energy emission rate calculated with this methodology and it is somewhat different from the one found in the literature (Bisnovatyi-Kogan, G.S. 2010 and its references). Where $\eta_{i p}$ and $\eta_{m p}$ are the instantaneous and mean densities of the ${ }^{1} \mathrm{H}$, $\rho_{c}$ is the stellar central density, $\mathrm{E}_{p p}$ is the emitted energy and $\mathrm{R}_{p p}$ is the thermonuclear reaction rate for the ${ }^{1} \mathrm{H}$ in the stellar interior. 


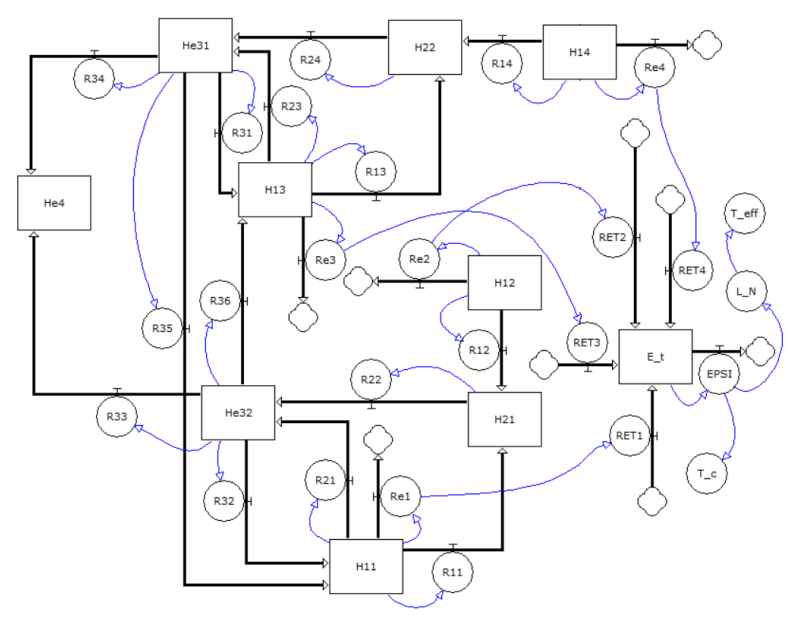

Figure 4 - Forrester diagram for PPC. In this figure are the state variables H12, H13, H14, H21, H22 \& E_t with their respective inflow and outflow rates (circles with letters $\mathrm{R}$ ) and variables EPSI, T_eff, T_C and $\mathrm{L}_{-} \mathrm{N}$ calculated in this work.

EPSI is the energy emission rate in the stellar interior, this rate is obtained from state variable E_t (accumulated total energy in the stellar interior) and rates RET1, RET2, RET3 and RET4 such as it is observed in the FD. The equation (8) is solved using the numerical method of Runge Kutta with an integration time of 0.01 and it is used to calculate the nuclear luminosity L_N, T_eff and central temperatures T_C of the star. The results for these variables are shown in Table 1 and normalized to solar values in Figure 5. Therefore, as it can be observed, our results are similar to those found in the literature.

$$
E P S I=\frac{E_{-} t}{d t}-(e 1+e 2+e 3+e 4)=-4\left(\frac{\eta_{i p}}{\eta_{m p}}\right) \frac{E_{p p}}{\rho_{c}} R_{p p} .
$$

Table 1 - Comparations with literature. The third co lumn has the solar mean values calculated from BisnovatyiKogan, G.S. (2010) and Kippenhahn, R., Weigert, A. \& Weiss (2012).

\begin{tabular}{|lcc|}
\hline \hline Parameter & This work & Literature \\
\hline T_c $(\mathrm{K})$ & $2.13 \times 10^{7}$ & $1.50 \times 10^{7}$ \\
T_eff $(\mathrm{K})$ & 5604 & 5777 \\
L_N $(\mathrm{W})$ & $3.66 \times 10^{26}$ & $3.85 \times 10^{26}$ \\
EPSI $(\mathrm{W} / \mathrm{Kg})$ & $1.48 \times 10^{-3}$ & $1.74 \times 10^{-3}$ \\
\hline \hline
\end{tabular}

Our effective temperature obtained is very near to value from observational atmospheric models, for energy emission rate and luminosity the situation is similar. Our central temperature indicate one a slightly difference that can be explained by fact that Hydrogen mass fraction was not analyzed.

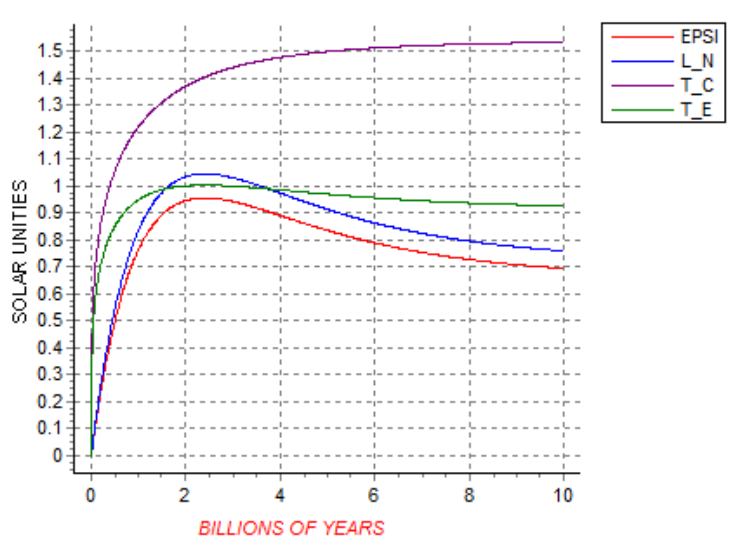

Figure 5 - EPSI, L_N, T_C \& T_E (being T_eff in FD) for a solar-type star. These results are normalized relative to solar mean values from Table 1 .

The Equations (9)-(12) represent the production of different chemical elements and the feedback inside the stellar interior for solar-type stars. These facts are consequence of the interactions between the particles which in turn are observable due to ST's perspective.

$$
\begin{gathered}
\frac{d \eta(p)}{d t}=-4 \frac{\eta_{i p}}{\eta_{m p}} R_{p p}-2 \frac{\eta_{i d}}{\eta_{m d}} R_{p d}-\frac{\eta_{i w}}{\eta_{m w}} R_{p w}+2 \frac{\eta_{i y}}{\eta_{m y}} R_{y y} \\
\frac{d \eta(d)}{d t}=2 \frac{\eta_{i p}}{\eta_{m p}} R_{p p}-\frac{3}{2} \frac{\eta_{i d}}{\eta_{m d}} R_{p d}, \\
\frac{d \eta(y)}{d t}=\frac{3}{2} \frac{\eta_{i p}}{\eta_{m d}} R_{p d}-3 \frac{\eta_{i y}}{\eta_{m y}} R_{y y}-\frac{\eta_{i z}}{\eta_{m z}} R_{y z} \\
\frac{d \eta(z)}{d t}=2 \frac{\eta_{i y}}{\eta_{m y}} R_{y y}-\frac{\eta_{i z}}{\eta_{m z}} R_{y z}+\frac{\eta_{i w}}{\eta_{m w}} R_{p w}
\end{gathered}
$$

where $\eta(p), \eta(d), \eta(y)$ and $\eta(z)$ are the densities of ${ }^{1} \mathrm{H},{ }^{2} \mathrm{H},{ }^{3} \mathrm{He}$ and ${ }^{4} \mathrm{He}$ respectively. The thermonuclear rate terms $\mathrm{R}$ with subscripts $p d, y y, y z \& p w$ refer to interactions ${ }^{1} \mathrm{H}-{ }^{2} \mathrm{H},{ }^{3} \mathrm{He}-{ }^{3} \mathrm{He},{ }^{3} \mathrm{He}-{ }^{4} \mathrm{He}$ and ${ }^{1} \mathrm{H}-{ }^{7} \mathrm{Li}$ respectively. The instantaneous and mean densities with subscripts $d, y, z, \& w$ represent the densities for ${ }^{2} \mathrm{H},{ }^{3} \mathrm{He}$, ${ }^{4} \mathrm{He}$ and ${ }^{7} \mathrm{Li}$ respectively.

\section{Modeling the galactic evolution process}

The Formation and evolution of our Milky Way is an open problem, due to the complexity of the feedback processes that arise from the energetic activity of massive or dying stars. This activity is taking place through winds, ionizing photons and Supernova explosions. These feedback processes involve a large range of scales and masses, from the subpicosecond scale of star formation to kiloparsecs scale of galactic winds, and from 1 to $10^{12} M_{\odot}$ or more. The simplest model of galactic evolution is a closed box in which variables such as hot gas, cold gas and stars have been modeled without interaction with the accretion from intergalactic medium (Monaco, P. 2004). In this work we 
have done a more detailed modelling of these variables and we have opened the system in order to consider the interaction with the intergalactic gas. Differently from PPC, the theory of the galaxy evolution is based on observations that show a qualitative behavior of the variables into the phenomenon. In this system the feedback loops are formed by interactions between variables that confirm the definition of dynamical system as a set formed by parts interacting. Galactic evolution involves large-scale treatment and its feedback loops contain unknown and known variables.

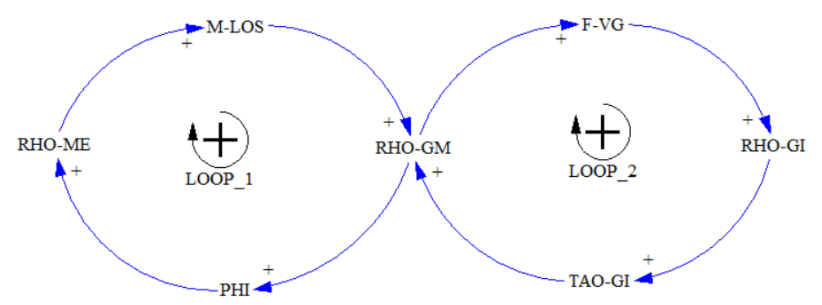

Figure 6 - Influence diagram for galactic evolution. The two feedback loops LOOP_1 and LOOP_2 are positive.

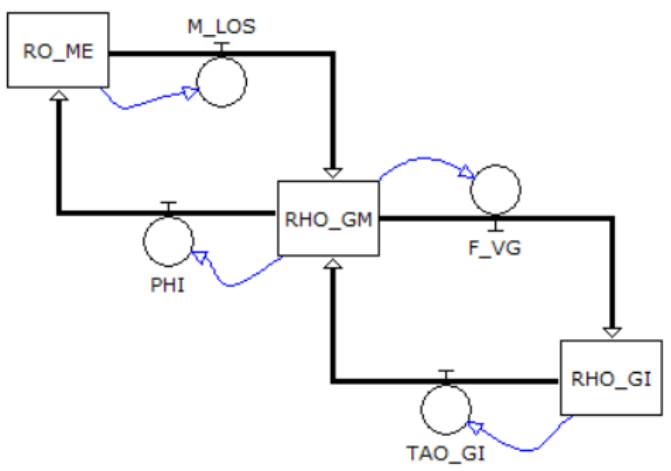

Figure 7 - Forrester diagram for galactic evolution process.

In the influence diagram, Figure 6 , we have two feedback loops which are responsible of the evolution of galaxies like our Milky Way (disc galaxies). The first feedback loop is formed by: stellar mass (RHO_ME), mass loss rate (M_LOS), density of molecular gas (RHO_GM) and stellar formation (PHI). This loop is linked to a second loop by means of RHO_GM. So, this second feedback loop is formed by: galactic wind (F_VG), density of intergalactic gas (RHO_GI) and infall of gas (TAO_GI). All variables in loop 2 can be considered unknown because there are no mathematical descriptions available in the literature. This lack of data and consistent equations makes the phenomenology of the galactic evolution more complex.

Forrester diagram for the galactic evolution process is shown in the Figure 7. The variables RHO_GI, RHO_GM and RHO_ME were classified as state variables whereas RHO_PM, TAU_GI, PHI, and RHO_VG as rates. This classification comes from the observational evidence because the density of molecular gas (RHO_GM) needed to form stars depends on: the fall of gas (TAO_GI) and mass loss (RHO_PM). TAO_GI come from intergalactic space and RHO_PM from stars. In turn, one part of the gas, into the molecular cloud, will form stars (PHI) and another part will feed to the galactic wind (F_VG). The stellar formation represented by (RHO_ME) depends on formation rate PHI (found in the literature as stellar formation rate). We have associated to this state variable the rate RHO_PM that represents the mass loss suffered by the stars in its evolutionary process. The density of intergalactic gas RHO_GI depends on the galactic wind RHO_VG, one part of this gas (TAO_GI) will fall into the galaxy due to the gravitational interaction. The qualitative mathematical model for galactic evolution process obtained from FD is given by equations:

$$
\frac{d \rho_{g m}}{d t}=\left(M_{l o s s}+\tau_{g i}\right)-\left(\phi+\rho_{v g}\right)
$$

$$
\begin{gathered}
\frac{d \rho_{m e}}{d t}=\phi-M_{l o s}, \\
\frac{d \rho_{g i}}{d t}=\rho_{v g}-\tau_{g i},
\end{gathered}
$$

where PHI is $\phi$, RHO_GM is $\rho_{g m}$, RHO_GI is $\rho_{g i}$, RHO_ME is $\rho_{m e}$, TAO_GI is $\tau_{g i}, \mathrm{~F}_{-} \mathrm{VG}$ is $\rho_{v g}$ and M_LOS is $\mathrm{M}_{\text {los }}$.

The equations (13)-(15) show that $\rho_{g m}$ has a decreasing rate because it will be transformed in stars while $\rho_{m e}$ is increasing because the majority of gas inside the molecular cloud will be used to form stars. The $\rho_{g i}$ is showing a similar behavior to $\rho_{m e}$ but maintaining low values because the gas in the intergalactic space is less important than the gas inside molecular cloud and than the gas transformed in stars. This behavior resulting from equations (13)-(15) is illustrated in Figure 8. Similar to PPC's simulation, the numerical solutions of the equations (13) to (15) is obtained using numerical method of Runge Kutta with an integration time of 0.01 . Our results have a qualitative behavior similar to the figures of Monaco, P. (2004).

Is necessary to say that the Milky Way may merge or interact with another galaxy, causing a significant boost or suppresion of all these processes (Lehner, N. \& Howk, J.C. 2011 and Lehner, N. et al. 2012). This was not still included in our simulation. Another factor is the Dark Cold Matter, our model still no has the effects of this type of matter included. 


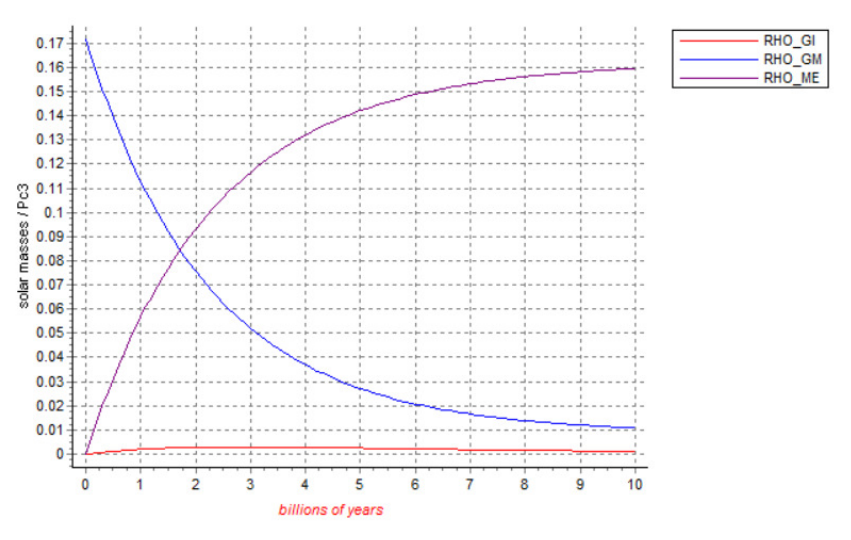

Figure 8 - Results for galactic evolution process. Solutions for variables RHO_GI, RHO_GM and RHO_ME were obtained via numerical integration from equations (13) to (15).

\section{Discussions and conclusions}

In this work we have applied SD to two astronomical phenomena, one of these is a problem very well understood and with a lot of results in the literature for its testing, the PPC. In the another case, we have modeled a problem whose information is much more limited but we have obtained qualitative behavior that can be compared to theory/observations, the galactic evolution process. In the first case we confirmed with SD we obtained similar results to literature (Table 1 and Figure 5) and in the second case we propose to use SD to understand a complex phenomenon like galactic evolution. Furthermore, SD offers different representations like state variables, rates, exogenous variables and delays that complemented with an appropriate classification made possible a consistent modeling and simulation process based on the theory and/or observations.

The collisions between ${ }^{1} \mathrm{H}$ atoms, that generate the others chemical elements, are the reflex of the dynamics of the stellar interior. This dynamic can be expressed in terms of the feedback loops such as is enlighttened in Figure 3 and confirmed by the linear differential equations (9)-(12). In solar-type stars, ${ }^{1} \mathrm{H}$ is the catalyst element in the reaction because it generates the two feedback loops. Therefore, we confirm one of the premises of ST: feedback as responsible of the dynamical behavior of the system. Feedback is responsible of the dynamic, but also it keeps the stellar stability. The ${ }^{1} \mathrm{H}$ feedback produces the nuclear energy to counter the gravitational contraction and generates stability in the stellar evolution. In the case of low-mass stars, the time calculated on the main sequence is nearly $10^{9}$ years that corresponds to the ${ }^{1} \mathrm{H}$ burning time duration in the stellar interior. From main sequence phase the stars evolve to different states of instability.

This work shown that through the SD is possible to obtain a mathematical model consistent with the theory and/or observations and therefore SD can be an important methodological tool in some problems where the theory is totally or partially unknown. This is the case of galactic evolution, where exist feedback loop correlations between elements unknown and partially known. Mathematical modeling done by means of traditional forms is very complicated, while in our case, owing to the SD's representations, we obtained a set of linear equations that describe causal relationships and the results are consistent with available observations. In fact, the phenomenological complexity of galactic evolution is presented in a more understandable due to the clarification of the correlations between these variables.

Due to results cited above and to the fact that in Astronomy there are many cases of complex systems whose dynamical behavior is determined by the feedback loops, the authors are applying system dynamics in some of these astronomical phenomena.

\section{References}

[1] Ade, P., Aghanim, N., Armitage-Caplan, C., Arnaud, M., et al. Planck 2013 results. XVI. Cosmological parameters. DOI:10.1051/0004-6361/201321591.

[2] Andrade, H., Ramirez, A., Ulloa, A. \& Ulloa, S. System Dynamics applied to Analysis Modeling and Simulation of Chain Growth Polymerization Process, International conference on System Dynamics, System Dynamics Society, $12^{\text {th }}$, Stirling, Codfish (1994).

[3] Andrade, H. Sotaquirá, R., Dynner, I., Espinoza, A. \& Lopez, H. Pensamiento Sistémico: Diversidad en Busqueda de Unidad, Ediciones UIS, Bucaramanga/Colombia, (2001).

[4] Bisnovatyi-Kogan, G.S. Stellar Physics 2: Stellar Evolution and Stability, Springer, Berlin/Germany, (2010).

[5] Catelan, M. Structure and Evolution of Low-Mass Stars: An Overview and Some Open Problems, AIP Conference Proceedings, Volume 930, pages 39-90 (2007). DOI:10.1063/1.2790333.

[6] Clayton, D. Principles Nucleosynthesis and Evolution Stellar, University of Chicago press edition, Chicago/USA, 1983.

[7] Ford, A. Modelling the Environments: an introduction to system dynamics modelling of environmental system, Island press: Washington D.C., USA, (1999).

[8] Forrester, J. Industrial dynamics, Martino Fine Books, Portland/USA, (1961, 2013).

[9] Forrester, J. Urban dynamics, Pegasus Communications, Portland/USA, (1969).

[10] Forrester, J. Lessons from System Dynamics Modeling, System Dynamics Review, volume 3, No. 2, pages 136-149, (1987). DOI:10.1002/sdr.4260030205.

[11] Forrester, J. System dynamics, systems thinking, and soft OR, System Dynamics Review, volume 10, pages 245-256, (1994). DOI:10.1002sdr.4260100211. 
[12] Forrester, J. Economic theory for the new millennium, System Dynamics Review, volume 29, No.1, pages 2641, (2013). DOI:10.1002/sdr.1490.

[13] Kippenhahn, R., Weigert, A., \& Weiss, A. Stellar Structure and Evolution. Astronomy and Astrophysics library. Springer Berlin, Heidelberg/Germany, (2012).

[14] Lehner, N. \& Howk, J. C. A Reservoir of Ionized Gas in the Galactic Halo to Sustain Star Formation in the Milky Way, Science, Volume 334, pages 955, (2011). DOI:10.1126/science.1209069.

[15] Lehner, N.; Howk, J. C.; Thom, C.; Fox, A. J.; Tumlinson, J.; Tripp, T. M. \& Meiring, J. D. High-velocity clouds as streams of ionized and neutral gas in the halo of the Milky Way, Monthly Notices of the Royal Astronomical Society, Volume 424, pages 2896-2913, (2012). DOI:10.1111/j.1365-2966.2012.21428.x.
[16] Monaco, P. Physical regimes for feedback in galaxy formation, Monthly Notices of the Royal Astronomical Society, Volume 352, pages 181-204, (2004). DOI:10.1111/j.1365-2966.2004.07916.x.

[17] Parker, P., Bahcall, J.N. \& Fowler, W.A. Termination of the Proton-Proton Chain in Stellar Interiors, Astrophysical Journal, volume 139, page 602-621, (1964). DOI:10.1086/147789.

[18] Schwarzschild, M. Structure and evolution of the stars, Dover publications INC, New York/USA, (1955).

[19] Sterman, J. Business Dynamics: Systems Thinking and Modeling for a Complex World, McGraw-Hill Education, Lexington/USA, (2000). 